\title{
Development and Utilization of Space Fission Power Systems
}

\author{
Michael G. Houts \\ NASA Marshall Space Flight Center, MSFC, AL, 35812 \\ Lee S. Mason ${ }^{2}$ and Donald T. Palac ${ }^{3}$ \\ NASA Glenn Research Center, Cleveland, OH, 44135 \\ and \\ Scott E. Harlow ${ }^{4}$ \\ US Department of Energy, Germantown, MD, 20874
}

\begin{abstract}
Space fission power systems could enable advanced civilian space missions. Terrestrially, thousands of fission systems have been operated since 1942. In addition, the US flew a space fission system in 1965, and the former Soviet Union flew 33 such systems prior to the end of the Cold War. Modern design and development practices, coupled with 65 years of experience with terrestrial reactors, could enable the affordable development of space fission power systems for near-term planetary surface applications.
\end{abstract}

\section{Nomenclature}

$\begin{array}{ll}\text { DOE } & =\text { Department of Energy } \\ \text { ETDP } & =\text { Exploration Technology Development Program } \\ \text { FSP } & =\text { Fission Surface Power } \\ \text { FSPS } & =\text { Fission Surface Power System } \\ \text { INSRP } & =\text { Interagency Nuclear Safety Review Panel } \\ \text { ISS } & =\text { International Space Station } \\ \text { NaK } & =\text { Sodium-Potassium eutectic } \\ \text { NASA } & =\text { National Aeronautics and Space Adminstration } \\ P C A D & =\text { Power Control and Distribution }\end{array}$

\section{Introduction}

Zission surface power (FSP) systems could be used to provide power anytime, anywhere on the surface of the Moon or Mars. FSP systems could be used at polar locations, at locations away from the poles, or in permanently shaded regions, with excellent performance at all sites. A potential reference $40 \mathrm{kWe}$ option has been devised that is cost-competitive with alternatives while providing more power for less mass anywhere on the lunar surface. The reference FSP system (FSPS) is also readily extensible for use on Mars. At Mars the system would be capable of operating through global dust storms and providing year-round power at any Martian latitude.

Under the NASA Exploration Technology Development Program (ETDP), NASA and the Department of Energy (DOE) have begun technology development on Fission Surface Power (FSP). The primary customer for this technology is the NASA Constellation Program which is responsible for the development of surface systems to support human exploration on the moon and Mars. The objectives of the FSP technology project are:

1) Develop FSP concepts that meet expected surface power requirements at reasonable cost with added benefits over other options.

2) Establish a hardware-based technical foundation for FSP design concepts and reduce overall development risk.

\footnotetext{
${ }^{1}$ Nuclear Research Manager, Exploration Advanced Capabilities Office/VP33, Member.

${ }^{2}$ Principal Investigator, Fission Surface Power, Power and Electric Propulsion Division/RPT0, Member.

${ }^{3}$ Project Manager, Fission Surface Power, Power and Electric Propulsion Division/RPT0, Member.

${ }^{4}$ Lead, Space Reactors, Radioisotope Power Systems, NE-34.
} 
3) Reduce the cost uncertainties for FSP and establish greater credibility for flight system cost estimates.

4) Generate the key products to allow Agency decision-makers to consider FSP as a viable option for flight development.

In order to achieve these goals, the FSP project has two main thrusts: Concept Definition and Risk Reduction. Under Concept Definition, NASA and DOE are performing trade studies, defining requirements, developing analytical tools, and formulating system concepts. A typical FSPS consists of the reactor, shield, power conversion, heat rejection, and power conditioning and distribution (PCAD). Studies are performed to identify the ideal design parameters for each subsystem that allow the system to meet the requirements with reasonable cost and development risk. Risk Reduction provides the means to evaluate technologies in a laboratory test environment. Hardware prototypes are built and operated to verify performance expectations and resolve design uncertainties.

The Concept Definition and Risk Reduction activities are highly coupled and the products are staggered so that the results of one can influence the other. For example, data from an electromagnetic pump test could be used to anchor a reactor thermal-hydraulic code that is used to design the flight primary heat transport circuit. That design could then be used to form the basis for a higher fidelity ground test loop. Details of the reference FSPS are given in Reference 1, and details of the FSPS technology development program are given in Reference 2.

\section{Design for Reliability and Affordability}

The primary design consideration for any space system is safety. Following safety; however, different design considerations dominate depending on the specific system or application.

Once safety criteria are met, the design of FSP systems is dominated by the need for reliability and affordability. While performance is always a consideration for space systems, the inherent performance of FSP systems in the power range of interest $(40 \mathrm{kWe})$ is such that maximizing performance is not a design driver. The significant performance advantage of FSP systems for providing steady-state power in the $40 \mathrm{kWe}$ range is primarily due to the independence of FSP systems from available sunlight. This allows FSP systems to readily provide full power during extended periods of darkness (lunar night) or drastically reduced sunlight (Mars global dust storms).

To help ensure both reliability and affordability, a reference FSP concept has been chosen that draws heavily on US and international terrestrial reactor experience. The reference concept uses 316 stainless steel as the primary structural material and fuel clad, and uranium dioxide as the fuel form. This combination of fuel, clad, and structural material has been used in terrestrial reactors throughout the world, with thousands of years of cumulative operating experience.

A pumped alkali metal $(\mathrm{NaK})$ loop is the reference method for transferring power from the reactor core to the power conversion subsystem. Pumped NaK loops were used to transfer thermal power in all 34 of the space fission systems flown to date. In addition to space reactor experience in the thermal power range of interest, alkali metal loops have also been used to cool terrestrial reactors that operated for decades at significantly higher power levels.

By space standards the reference $40 \mathrm{kWe}$ FSPS is high power. However, by terrestrial standards the reference FSPS is extremely low power. Terrestrial reactors routinely operate at thermal power levels 20,000 times higher than those required by the reference FSPS, and alkali metal cooled systems have been operated at thermal power levels over 2000 times higher. The relatively low operating power of the FSPS creates a relatively benign nuclear operating environment. For the eight year design life, fuel burnup is an order of magnitude lower than that routinely seen in terrestrial systems. This helps provide high confidence in the fuel system. Fast neutron fluence (primary source of radiation damage) is two orders of magnitude lower than that endured in some terrestrial reactors. This helps provide high confidence in the performance of reactor materials. Designing the system to operate well within established operating envelopes improves reliability and affordability.

The reference FSPS uses Stirling engines to convert heat to electricity. Stirling engines based on similar technology are being flight qualified for use with radioisotope power systems. Stirling engines are also being used for a variety of terrestrial applications, including cogeneration. A key attribute of Stirling power conversion is that it allows for high thermal-to-electric power conversion efficiency $(>20 \%)$ while maintaining modest peak system operating temperatures and a manageable radiator size. Power Control and Distribution (PCAD) and radiator 
technologies are based on previous terrestrial and space experience, including the International Space Station (ISS). A potential layout of the reference FSPS (excluding radiator) is shown in Figure 1. An artist's rendition of the FSPS emplaced on the lunar surface is shown in Figure 2.

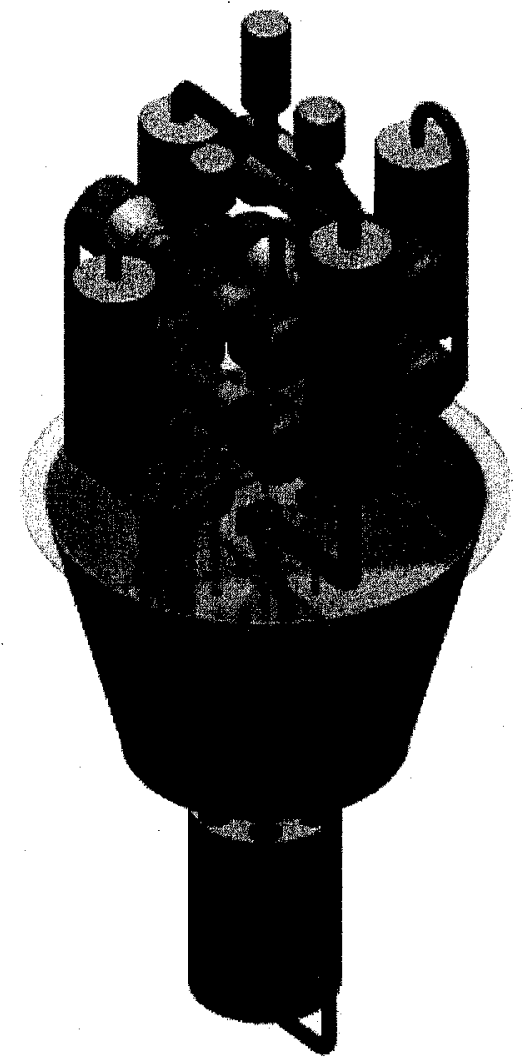

Figure 1. Reference FSPS layout (courtesy Lou Qualls, Oak Ridge National Laboratory)

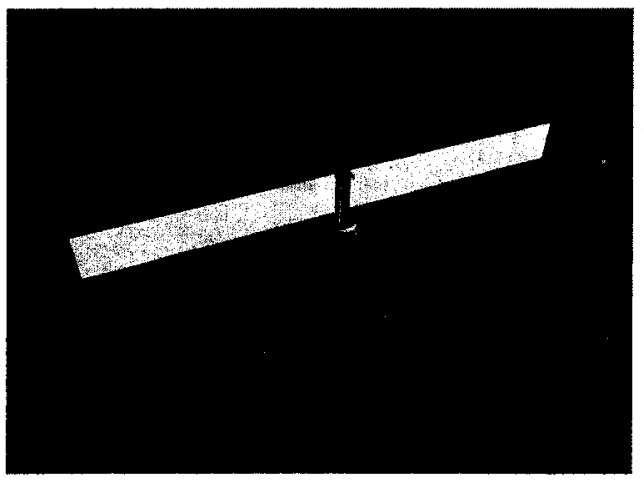

Figure 2. Reference FSPS emplaced on lunar surface (courtesy Lee Mason, Glenn Research Center)

American Institute of Aeronautics and Astronautics 


\section{Development and Flight Qualification}

The development and qualification of an FSPS can be optimized to ensure reliability and affordability. As a precursor to a potential flight development program, a project to develop FSP technology has been initiated. The FSPS technology development project is managed by Glenn Research Center (GRC) in Cleveland, OH. GRC is also responsible for power conversion, heat rejection, and PCAD development, referred to as the balance-of-plant. Marshall Space Flight Center (MSFC) in Huntsville, AL provides expertise in reactor simulation, primary loop components and non-nuclear testing. The DOE is represented by four National Labs: Idaho, Los Alamos, Oak Ridge, and Sandia. Each brings unique capabilities to the team. Some of the key contributions of the DOE Labs are in the areas of reactor and shield design, modeling and tools, fuel development, instrumentation and control, materials and irradiation testing, heat exchangers, and pumps. Various industry partners and university participants also play key roles in the technology development.

Early risk reduction efforts are focused on demonstrating component technologies under expected FSP conditions, using modified commercial hardware. At MSFC, testing has been completed on an annular linear induction pump (ALIP) that was formerly developed for the Experimental Breeder Reactor-II (EBR-II). Idaho National Lab removed the pump from storage and refurbished it for use in MSFC's NaK primary test circuit. Test data is now available for use in system design studies and for designing more flight-prototypic ALIPs. At GRC, a pair of opencycle commercial Brayton micro-turbines were modified and assembled into a dual, closed-Brayton cycle power system to produce upwards of $30 \mathrm{kWe}$. A pair of commercial, $1 \mathrm{kWe}$ free-piston Stirling power units are currently being modified at GRC with a NaK hot-end heat exchanger for testing at MSFC in late 2008. GRC has also tested several heat pipe radiator prototypes that will serve as a point-of-departure for a full-scale Radiator Demonstration Unit planned for thermal-vacuum testing in 2009. The centerpiece of the risk reduction activities is the non-nuclear Technology Demonstration Unit (TDU) planned for completion in 2013. The goal of the TDU is to assemble the major components of the FSPS (heat source, power conversion, heat rejection, and PCAD) and conduct non-nuclear, integrated system testing in thermal-vacuum to evaluate overall performance. Plans are to develop full-scale components and assemble a 1/4th power (10 to $12 \mathrm{kWe}$ ) system in GRC's Vacuum Facility \#6. DOE and MSFC will jointly develop the reactor simulator. GRC and its industrial partners will develop the balance-of-plant. The testing will help to verify performance projections, develop safe and reliable control methods, and gain valuable systems operating experience while providing a tangible and measurable technology milestone.

A flight development program could potentially follow the technology development program. That program would entail an optimal combination of nuclear and non-nuclear development and testing. Nuclear testing would include both cold and warm nuclear criticals to experimentally verify calculated parameters used in safety analyses and in devising specific operating procedures. Additional nuclear testing would be performed to verify the performance of both in-core and ex-core components in the FSP environment. Non-nuclear testing would be used in the development and qualification of components, subsystems, and the integrated system. Non-nuclear testing is used extensively in the development of terrestrial nuclear systems, and is in some ways even more applicable to the development of FSP systems because of the relatively benign nuclear environment experienced by those systems.

\section{FSP Launch, Emplacement, and Operation}

FSP systems would be essentially non-radioactive at launch, because the actual flight units would not be operated at significant power for long periods of time prior to launch. However, the FSPS would still be considered a "nuclear" launch, and the launch would be approved by the Office of the President via the Interagency Nuclear Safety Review Panel (INSRP) process. The INSRP has provided reviews of all nuclear powered spacecraft launched by the United States, and the INSRP process could be readily applied to FSP systems.

Radiation would be emitted from the FSPS during operation on the lunar surface. The FSPS could be designed to either carry all required radiation shielding from earth, or to use a combination of shielding brought from earth and shielding from in-situ materials (e.g. regolith). FSP shielding is typically designed such that the radiation dose rate from the FSPS would be less than $10 \%$ of the radiation dose rate from natural background sources at the habitat boundary. Shielding could be used to further adjust the dose rate from the reactor as desired, with a corresponding mass savings or mass penalty. During operation there would be no radiation-related exclusion zone around the 
FSPS, although it could be desirable to minimize operations in very close proximity to the FSPS for a variety of reasons (e.g. dust mitigation, electrical hazards, minimizing radiation dose).

FSPS start could occur within hours of landing on the lunar surface, or after an extended period of time, depending on mission requirements. For emplaced options (e.g. regolith shielding) time would be required to position the FSPS and complete any required regolith moving. System start is accomplished by rotating control drums a fraction of a turn to increase reactivity relative to the launch configuration. Control drums are then adjusted until the reactor is providing hot working fluid $(\mathrm{NaK})$ at the desired core outlet temperature, which is $\sim 880 \mathrm{~K}$ for the reference FSPS. The FSP reactor is inherently load following, and will naturally maintain a near-constant temperature independent of the amount of power being extracted from the core. As uranium fuel is consumed, the $\mathrm{NaK}$ outlet temperature would naturally decrease to compensate for lost reactivity. To avoid a reduction in efficiency caused by reduced $\mathrm{NaK}$ outlet temperature, reactivity would periodically be inserted by adjusting one or more of the control drums. Although the system could run for a long period of time without such adjustments, it may be desirable to adjust the drums every 1-2 months to maintain operating temperature within a narrow band $(\sim 5$ $\mathrm{K})$.

There are no fundamental life-limiters for the FSPS. The reference FSPS will consume $\sim 1 \mathrm{~kg}$ of uranium every 15 years, and under nominal conditions would have enough reactivity to run for decades. However, it is very difficult to qualify space systems for extremely long life, and because of that a reference lifetime requirement of 8 years was chosen. It is likely that if power was still required after 8 years and the FSPS was operating as expected, it would continue to be used.

After shutdown, radiation emitted by the FSPS would decrease rapidly. If desired, a replacement FSPS could be emplaced at the same site (within a few meters of the original system), and potentially use some of the same infrastructure (e.g. cabling).

\section{Conclusion}

The relatively modest mission requirements for FSP, coupled with over six decades of terrestrial reactor experience, would enable a reliable, affordable FSPS to be developed. Ongoing technology development work will enable an informed decision to be made as early as 2013 on whether or not to proceed with flight development of an FSPS.

\section{Acknowledgements}

The work described in this paper is being funded by NASA ETDP. Any opinions expressed are those of the authors, and do not necessarily represent those of ETDP.

\section{References}

${ }^{1}$ Mason, L. S., “AFSPS Reference Power System Concept," Space Technology \& Applications International Forum (STAIF2008), AIP CPXXX, American Institute of Physics, Albuquerque, NM, 2008, pp.

${ }^{2}$ Palac, D. T., "AFSPS Technology Program," Space Technology \& Applications International Forum (STAIF-2008), AIP CPXXX, American Institute of Physics, Albuquerque, NM, 2008, pp. 